OPEN ACCESS

Edited by:

Liangcai Zhang,

Janssen Research and Development,

United States

Reviewed by:

Keshen Li,

Jinan University, China

Liang Cheng,

Harbin Medical University, China

*Correspondence:

Nan Zhang

nkzhangnan@yeah.net

Wei Jiang

jiangwei_med@163.com

${ }^{t}$ These authors have contributed

equally to this work

Specialty section:

This article was submitted to Statistical Genetics and Methodology,

a section of the journal

Frontiers in Genetics

Received: 28 May 2019

Accepted: 08 July 2020

Published: 11 August 2020

Citation:

Wang Y, Gao L, Lang W, Li H, Cui P, Zhang $N$ and Jiang W (2020)

Serum Calcium Levels and Parkinson's Disease: A Mendelian

Randomization Study.

Front. Genet. 11:824.

doi: 10.3389/fgene.2020.00824

\section{Serum Calcium Levels and Parkinson's Disease: A Mendelian Randomization Study}

\author{
Yanchao Wang ${ }^{1,2+}$, Luyan $\mathrm{Gao}^{3+}$, Wenjing Lang ${ }^{1}, \mathrm{He} \mathrm{Li}{ }^{1}$, Pan Cuil, Nan Zhang ${ }^{1 *}$ and \\ Wei Jiang ${ }^{1 *}$ \\ ${ }^{1}$ Department of Neurology, Tianjin Neurological Institute, Tianjin Medical University General Hospital, Tianjin, China, \\ ${ }^{2}$ Department of Neurology, Affiliated Hospital of Chifeng University, Chifeng, China, ${ }^{3}$ Department of Neurology, The Fourth \\ Central Clinical College of Tianjin Medical University, Tianjin, China
}

Background: Though increasing epidemiological studies have evaluated the correlation between serum calcium contents and Parkinson's disease (PD), the results are inconsistent. At present, whether there is a causal association between serum calcium content and PD remains undetermined.

Objective and Methods: This study was designed to explore the relationship between increased serum calcium contents and PD risk. In this present study, a Mendelian randomization trial was carried out using a large-scale serum calcium genome-wide association study (GWAS) dataset ( $N=61,079$, Europeans) and a large-scale PD GWAS dataset ( $N=8,477$, Europeans including 4,238 PD patients and 4,239 controls). Here, a total of four Mendelian randomization methods comprising weighted median, inversevariance weighted meta-analysis (IVW), MR-Egger, and MR-PRESSO were used.

Results: Our data concluded that genetically higher serum calcium contents were not significantly related to $\mathrm{PD}$.

Conclusion: In conclusion, we provided genetic evidence that there was no direct causal relationship between serum calcium contents and PD. Hence, calcium supplementation may not result in reduced PD risk.

Keywords: Parkinson's disease, serum calcium, Mendelian randomization, pleiotropy analysis, power analysis

\section{INTRODUCTION}

Previous studies indicated that calcium is involved in many biological processes, and altered calcium homeostasis is widely regarded as a basis for cognitive deficits in normal subjects and certain neurodegenerative diseases (LaFerla, 2002; Larsson et al., 2017). Parkinson's disease (PD) is the second most common neurodegenerative disorder, which affects $1-2 \%$ people older than 65 years (Gibrat et al., 2009; Saad et al., 2011; Liu et al., 2015a,b; Liu G. et al., 2016). Particularly, emerging epidemiological research has evaluated the correlation between serum calcium contents and PD, and calcium dysregulation has been found in PD (Abou-Raya et al., 2009; Meamar et al., 2013; Schapira, 2013; Liu J. et al., 2016). However, the results reported by these studies are often inconsistent.

Abbreviations: BMI, Body Mass Index; CI, confidence interval; GWAS, genome-wide association study; HDL, high density lipoprotein; IVW, inverse-variance weighted; MAF, Minimum allele frequency; MR, Mendelian randomization; MR-PRESSO, Mendelian randomization pleiotropy residual sum and outlier; OR, odds ratio; PD, Parkinson's disease; SD, standard deviation; SNP, single-nucleotide polymorphism. 
In 2009, Abou-Raya et al. (2009) evaluated the relationship between bone changes and PD. In their study, the bone density and mineral metabolism in 82 PD patients and 68 age- and sexmatched controls were measured, of which the results showed that serum calcium and vitamin D levels were significantly decreased in PD patients compared with controls. Similarly, Meamar et al. (2013) compared serum calcium contents from 105 PD patients and 112 matched controls in 2013, and indicated that serum calcium contents were significantly decreased in PD patients relative to controls. In addition, Liu J. et al. (2016) also found that serum calcium contents were significantly decreased in 77 PD patients with dementia compared to 75 healthy control subjects in 2016. It should be noted that no significant difference in serum calcium content was observed between the PD group without dementia and the healthy control group in their study.

At present, it remains unclear whether there is a causal relationship between serum calcium content and PD. Here, we tried to find the causality between the serum calcium levels and PD, thereby providing effective therapies for PD. Recently, Mendelian randomization methods have been widely applied to assess causal relationships through genome-wide association study (GWAS) datasets (Mokry et al., 2015; Nelson et al., 2015; Larsson et al., 2017; Manousaki et al., 2017; Cheng et al., 2018, 2019; Hu et al., 2018; Liu et al., 2018, 2019; Sun et al., 2019; Zhuang et al., 2019a,b). Therefore, a Mendelian randomization study was carried out to explore the genetic relationship between serum calcium content and PD through the large-scale serum calcium and PD GWAS datasets in our study.

\section{MATERIALS AND METHODS}

\section{Study Design}

A previous study reported that human genetic variants are randomly allocated, which is the basis of the Mendelian randomization method (Emdin et al., 2017). These genetic variants are mostly independent from confounding variables and can be regarded as instrumental proxies to assess the causal relationship between serum calcium levels and its outcome. Mendelian randomization was conducted according to three primary assumptions as described in the previous reports (Emdin et al., 2017; Larsson et al., 2017). Assumption (1): the selected genetic variants are related to serum calcium levels; assumption (2): these genetic variants are not related to confounders; assumption (3): these genetic variants affect PD risk only via serum calcium levels. Meanwhile, assumptions (2 and 3) were together regarded as independence from pleiotropic effects. Our present work was performed using the publicly available largescale GWAS dataset. Informed consent from all the participants was obtained in all the original research.

\section{Serum Calcium GWAS Dataset}

In this study, eight genetic variants influencing serum calcium content with genome-wide significance $(P<5.00 \mathrm{E}-08)$ were used as the instrumental proxies (O'Seaghdha et al., 2013). The dataset contained 39,400 subjects deriving from 17 populationbased cohorts in the discovery stage and 21,679 subjects in the replication stage ( $N=61,079$, Europeans). All the genetic variants were distributed at various genes and were not in linkage disequilibrium as presented in Table 1. The measurement of serum calcium content was described in details in Additional File 1.

\section{PD GWAS Dataset}

Discovery PD GWAS dataset was derived from a meta-analysis of five independent PD GWAS datasets $(n=8,477$, Europeans including 4,238 PD patients and 4,239 controls) (Pankratz et al., 2012). The relationship between each dataset and PD susceptibility was tested using a logistic regression model, and the results of each dataset were performed for meta-analysis. Here, we used the meta-analysis results of about 2,525,704 SNPs. Validation of the PD GWAS dataset was derived from a case control-associated map obtained from family history of disease in the UK Biobank (Liu et al., 2017). In brief, this GWAS dataset consisted of 4,627 PD cases and 109,826 controls, all of which are of European ancestry. Here, we used the genome-wide analyzed results by proxy. Informed consent from all the participants was obtained in all the original research. Link for the serum calcium and PD GWAS datasets is https://www.ebi.ac.uk/gwas/.

\section{Pleiotropy Analysis}

For Mendelian randomization studies, a crucial problem is potential contradiction of assumptions $(2,3)$ via pleiotropic effects which occur when a genetic instrument is related to the outcome, independent of the exposure. In this work, a pleiotropy evaluation was conducted to confirm that the eight genetic variants did not affect PD risk via biological pathways outside serum calcium content, and three steps were performed to mitigate the risk of pleiotropy.

In 2016, a major review from the Lancet Neurology reported that PD risk factors included body mass index (BMI), diabetes, blood cholesterol, hypertension, alcohol, vitamins, fat and other micronutrients, and PD protective factors included smoking, coffee, tea and serum urate (Ascherio and Schwarzschild, 2016). At stage 1, we evaluated the potential pleiotropy using some known confounders including major lipids (total cholesterol and triglyceride, low density lipoprotein, high density lipoprotein, etc.), type 2 diabetes, hypertension, blood pressure, BMI, waist circumference, hip circumference and waist-to-hip ratio, smoking, alcohol consumption, serum urate and vitamin D levels. The significance threshold for the relationship between the eight variants and the above-mentioned confounders was defined to be a Bonferroni-corrected $P<0.05 / 8=0.00625$.

Previous studies indicated that a statistical method, termed MR-Egger, could provide a reliable evaluation for the instrumental variable assumption, and check for the presence of potential pleiotropy (Dale et al., 2017; Tillmann et al., 2017). Therefore, at stage 2, the MR-Egger was used to assess potential pleiotropic relationships between the genetic variants and confounders.

At stage 3, a recently developed statistical method, Mendelian randomization pleiotropy residual sum and outlier (MRPRESSO) (Verbanck et al., 2018), was used to confirm the horizontal pleiotropic outliers. 
TABLE 1 | Characteristics of 8 genetic variants in serum calcium and PD GWAS datasets.

\begin{tabular}{|c|c|c|c|c|c|c|c|c|c|c|c|c|c|c|}
\hline \multirow[t]{2}{*}{ SNP } & \multirow[t]{2}{*}{ Chr } & \multirow[t]{2}{*}{ Nearby genes } & \multirow[t]{2}{*}{$\mathrm{EA}^{\mathrm{a}}$} & \multirow[t]{2}{*}{ NEA } & \multirow[t]{2}{*}{$\mathrm{EAF}^{\mathrm{b}}$} & \multicolumn{3}{|c|}{ Serum calcium GWAS } & \multicolumn{3}{|c|}{ Discovery PD GWAS } & \multicolumn{3}{|c|}{ Validation PD GWAS } \\
\hline & & & & & & Beta (mg/dL)c & $\mathrm{SE}^{\mathrm{c}}$ & $P$-value ${ }^{c}$ & Beta $^{d}$ & SE $^{d}$ & $P$-value ${ }^{d}$ & Beta $^{d}$ & SE $^{d}$ & $P$-value ${ }^{\mathrm{d}}$ \\
\hline rs780094 & 2 & GCKR & $\mathrm{T}$ & $\mathrm{C}$ & 0.42 & 0.017 & 0.003 & $1.30 \mathrm{E}-10$ & -0.0037 & 0.0332 & 0.911 & -0.0190 & 0.0233 & 0.4156 \\
\hline rs1550532 & 2 & DGKD & $\mathrm{C}$ & G & 0.31 & 0.018 & 0.003 & $8.20 E-11$ & -0.0411 & 0.0354 & 0.2458 & -0.0547 & 0.0246 & 0.0265 \\
\hline rs1801725 & 3 & CASR & $\mathrm{T}$ & $G$ & 0.15 & 0.071 & 0.004 & 8.90E-86 & 0.0425 & 0.046 & 0.3547 & 0.0421 & 0.0334 & 0.2052 \\
\hline rs10491003 & 10 & GATA3 & $\mathrm{T}$ & $\mathrm{C}$ & 0.09 & 0.027 & 0.005 & 4.80E-09 & -0.0427 & 0.0566 & 0.4501 & -0.0564 & 0.0399 & 0.1576 \\
\hline rs7336933 & 13 & DGKH/KIAA0564 & $G$ & $A$ & 0.85 & 0.022 & 0.004 & $9.10 \mathrm{E}-10$ & -0.0352 & 0.046 & 0.4441 & 0.0564 & 0.0321 & 0.0788 \\
\hline rs1570669 & 20 & CYP24A1 & G & A & 0.34 & 0.018 & 0.003 & $9.10 \mathrm{E}-12$ & -0.0303 & 0.0342 & 0.3757 & -0.0051 & 0.0240 & 0.8318 \\
\hline rs7481584 & 11 & CARS & G & A & 0.7 & 0.018 & 0.003 & $1.20 E-10$ & 0.0429 & 0.0365 & 0.24 & 0.0018 & 0.0251 & 0.9430 \\
\hline rs17711722 & 7 & VKORC1L1 & $\mathrm{T}$ & $\mathrm{C}$ & 0.47 & 0.021 & 0.003 & $2.80 \mathrm{E}-11$ & 0.0714 & 0.0331 & 0.03075 & 0.0266 & 0.0228 & 0.2483 \\
\hline
\end{tabular}

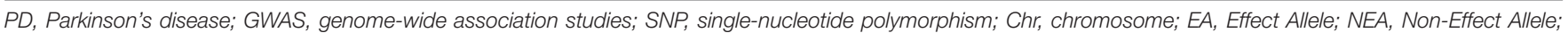

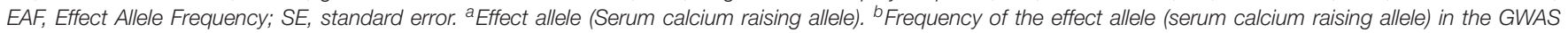

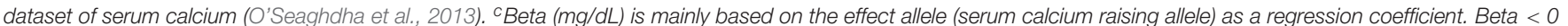

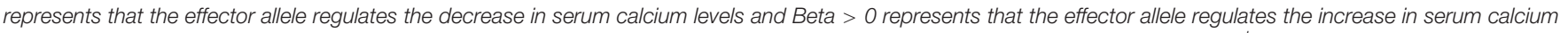

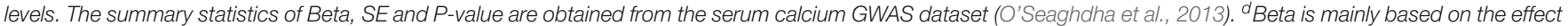

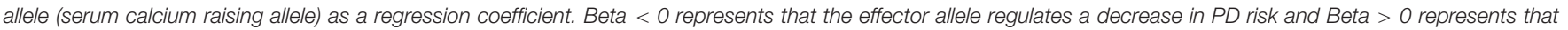

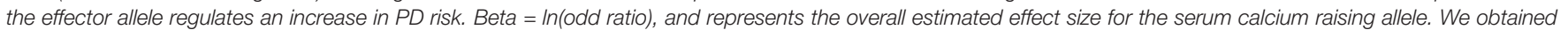
summary statistics of Beta, SE and P-value from the discovery and validation PD GWAS datasets of European descent (Pankratz et al., 2012; Liu et al., 2017).

\section{Mendelian Randomization Analysis}

In this study, Mendelian randomization analysis was conducted through four widely used methods, including MR-Egger, MRPRESSO, inverse-variance weighted meta-analysis (IVW) and weighted median (Burgess et al., 2017; Verbanck et al., 2018), which are based on different assumptions and are useful for examining the robustness with each other. The details for the MR-Egger, IVW and weightedmedian methods have been described in our previous study (Liu et al., 2018).

The IVW method assumed that all genetic variants conformed to the instrumental variable assumption, and the MR-Egger method assumed that these instrumental variables were not constant. A weaker hypothesis, the InSIDE assumption (Instrument Strength Independent of Direct Effect assumption), could still provide a consistent causal effect estimate (Bowden et al., 2015). In addition, the MRPRESSO method was proven to require a basic assumption that more than half of the genetic variants were qualified instrumental variables and satisfied both the balanced pleiotropy and InSIDE assumption (Verbanck et al., 2018). Finally, the weighted-median method can estimate a consistent causal effect when more than half of the genetic variants are effective instrumental proxies (Bowden et al., 2016). The odds ratio (OR) and $95 \%$ CI of PD corresponded to per $0.5 \mathrm{mg} / \mathrm{dL}$ elevation [1 standard deviation $(S D)]$ in serum calcium content. All analyses were performed through the R packages "MRPRESSO" (Verbanck et al., 2018) or "Mendelian Randomization" (Yavorska and Burgess, 2017).

For the statistical analysis of the genetic association of serum calcium content with PD risk, $P<0.05$ was considered as significant. In addition, a sensitivity analysis was conducted to evaluate the robustness of the causal estimates. Subsequently, each genetic variant was excluded one by one from the MR analysis via the leave-one-out permutation analysis, to assess the impact of each genetic variant on the causal estimates.

\section{Power Analysis}

Serum calcium variance $\left(R^{2}\right)$ proportion was assessed with the following formula:

$$
R^{2}=\sum_{i=1}^{K} \frac{\beta_{i}^{2} * 2 * M A F_{S N P_{i}}\left(1-M A F_{S N P_{i}}\right)}{\operatorname{var}(X)}
$$

Where $\beta_{i}$ is the effect size (beta coefficient) associated with the serum calcium content for $S N P_{i}, M A F_{S N P_{i}}$ is the minor allele frequency for $S N P_{i}, K$ represents genetic variant number, and $\operatorname{var}(X)$ represents serum calcium variance $\left(\operatorname{var}(X)=S D^{2}\right.$, and $1 S D=0.5 \mathrm{mg} / \mathrm{dL})$.

The instrumental variable strength (genetic variant related to serum calcium content) was assessed through the first-stage F-statistics. Generally, a threshold $(F>10)$ was used in case of bias in Mendelian randomization trials (Burgess and Thompson, 2011). Here, we calculated the F-statistics and statistical power using a web-based tool $\mathrm{mRnd}^{1}$ and a two-sided type-I error rate $\alpha$ of 0.05 (Brion et al., 2013).

\section{RESULTS}

\section{Association Between Serum Calcium Variants and PD}

From the discovery and validation PD GWAS datasets, eight genetic variants associated with serum calcium levels were extracted for statistical analysis. As shown in Table 1, the results suggested that all these genetic variants were not significantly related to $\mathrm{PD}(P>0.05 / 8=0.00625)$.

\section{Pleiotropy Analysis}

At stage 1, the results showed that rs780094 and rs1570669 variants were significantly related to some known confounders

\footnotetext{
${ }^{1}$ https://cnsgenomics.shinyapps.io/mRnd/
} 
$(P<0.05 / 8=0.00625)$. More specifically, rs780094 was significantly related to low density lipoprotein $(P=1.02 \mathrm{E}-07)$, high density lipoprotein $(P=2.67 \mathrm{E}-03)$, total cholesterol $(P=5.28 \mathrm{E}-41)$, triglyceride $(P=7.08 \mathrm{E}-125)$, type II diabetes $(P=1.00 \mathrm{E}-05)$, alcohol consumption $(P=3.65 \mathrm{E}-09)$, hip circumference $(P=3.40 \mathrm{E}-05)$ serum urate $(P=6.52 \mathrm{E}-39)$ and waist-to-hip ratio adjusted for BMI $(P=1.80 \mathrm{E}-03)$; rs 1570669 was significantly related to circulating vitamin $\mathrm{D}(P=5.33 \mathrm{E}-06)$. Hence, we excluded rs780094 and rs1570669 variants to meet the assumptions of the Mendelian randomization design. More detailed datasets were provided in Additional File 2. At stage 2, using the remaining six genetic variants (excluding rs780094 and rs1570669 variants identified at stage 1), the MR-Egger intercept test demonstrated that no evidence of pleiotropy was detected in the PD discovery GWAS dataset, with intercept $=0.000$ and $P=1.00$, or in the $\mathrm{PD}$ validation GWAS dataset, intercept $=-0.021$ and $P=0.562$. At stage 3 , no horizontal pleiotropic outliers were identified in either the discovery or validation PD GWAS datasets by MR-PRESSO. Hence, our Mendelian randomization trial focused on the remaining six genetic variants.

\section{Association Between Serum Calcium Content and PD Risk}

For the PD discovery dataset, the IVW analysis showed that per SD increase in serum calcium content $(0.5 \mathrm{mg} / \mathrm{dL})$ was not causally associated with a decreased $\mathrm{PD}$ incidence $(\mathrm{OR}=1.73$, 95\% CI: $0.47-6.37, P=0.408)$ using the remaining 6 genetic variants. Likewise, the weighted-median estimate $(\mathrm{OR}=1.72$, 95\% CI: 0.52-5.66, $P=0.374$ ), MR-Egger estimate ( $\mathrm{OR}=1.73$, 95\% CI: 0.12-25.9, $P=0.691)$ and MR-PRESSO estimate $(\mathrm{OR}=1.73,95 \% \mathrm{CI}: 0.47-6.37, P=0.446)$ did not show a significant causal association of serum calcium content with PD risk ( $P<0.05$ was considered as significance). For the $\mathrm{PD}$ validation dataset, our study demonstrated a similar conclusion that neither the IVW estimate (OR $=1.39,95 \% \mathrm{CI}: 0.45-4.31$, $P=0.564)$, weighted-median estimate $(\mathrm{OR}=1.78,95 \% \mathrm{CI}$ : 0.76-4.15, $P=0.185$ ), MR-Egger estimate ( $\mathrm{OR}=2.46,95 \% \mathrm{CI}$ : $0.25-23.9, P=0.437)$ or MR-PRESSO estimate $(\mathrm{OR}=1.39$, 95\% CI: $0.45-4.30, P=0.589)$ demonstrated that the increased serum calcium content was significantly related to PD risk. The details are shown in Table 2. Furthermore, the leave-one-out permutation analysis indicated the direction and precision of causal association between enhanced serum calcium content and increased PD risk was largely unchanged among these methods. In addition, we provide the individual estimates from different methods inferring the causality between each of serum calcium genetic variants and PD in the PD discovery and validation datasets, respectively (Additional Files 3, 4). The forest plot for MR estimates about the causal effect of genetically increased serum calcium levels on PD using IVW method is also added (Additional Files 5, 6).

\section{Power Analysis}

Here, all these six genetic variants could explain about $0.81 \%$ of the serum calcium variances $\left(R^{2}=0.81 \%\right)$. In both the
TABLE 2 | Mendelian randomization analysis using four methods.

\begin{tabular}{llllll}
\hline Dataset & Method & OR & SE & $\mathbf{9 5 \%} \mathbf{C l}$ & $\boldsymbol{P}$-value \\
\hline PD discovery dataset & Weighted_median & 1.72 & 0.61 & $0.52-5.66$ & 0.374 \\
PD discovery dataset & IWW & 1.73 & 0.66 & $0.47-6.37$ & 0.408 \\
PD discovery dataset & MR-Egger & 1.73 & 1.38 & $0.12-25.9$ & 0.691 \\
PD discovery dataset & MR-PRESSO & 1.73 & 0.66 & $0.47-6.37$ & 0.446 \\
PD validation dataset & Weighted_median & 1.78 & 0.43 & $0.76-4.15$ & 0.185 \\
PD validation dataset & IW & 1.39 & 0.58 & $0.45-4.31$ & 0.564 \\
PD validation dataset & MR-Egger & 2.46 & 1.16 & $0.25-23.9$ & 0.437 \\
PD validation dataset & MR-PRESSO & 1.39 & 0.58 & $0.45-4.30$ & 0.589 \\
\hline
\end{tabular}

SE, standard error; $O R>0$ and $O R<0$ means that high serum calcium levels increase and reduce the risk of PD. OR, odds ratio; Cl, confidence interval; $\mathrm{NW}$, Inverse-variance weighted meta-analysis. The association between serum calcium levels and $P D$ was at the significance level $P<0.05$.

PD discovery and validation GWAS datasets, the first-stage F-statistics for the instrument included six genetic variants with F more than 10, so there was no instrument bias. Our Mendelian randomization trial had $80 \%$ power to determine the impact size of moderate magnitudes with ORs $>=1.95$ and 1.48 per SD $(0.5 \mathrm{mg} / \mathrm{dL})$ increases in serum calcium content for PD risk in the PD discovery and validation GWAS dataset, respectively.

\section{DISCUSSION}

Calcium $\left(\mathrm{Ca}^{2+}\right)$ is a crucial second messenger that participates in a variety of cellular physiological processes. Particularly in neuronal cells, intracellular $\mathrm{Ca}^{2+}$ signaling is under extremely precise control to ensure the smooth functioning of many electrophysiological activities such as neuronal excitability, neurotransmitter secretion and synaptic plasticity. As a result, dysregulation of $\mathrm{Ca}^{2+}$ signaling is implicated in neurodegeneration disorders such as Alzheimer's disease, PD and Huntington's disease. Recently, the causality between genetically higher serum calcium and reduced $\mathrm{AD}$ incidence has been established (Jiang et al., 2018).

It has been well recognized that $\mathrm{Ca}^{2+}$ signaling abnormalities play a crucial role in $\mathrm{PD}$ pathogenesis. The coordinated regulation of $\mathrm{Ca}^{2+}$ fluxes is compromised in $\mathrm{PD}$, which induces selective toxicity in the dopaminergic neurons in the substantia nigra. PD risk is significantly reduced in hypertensive patients treated with L-type $\mathrm{Ca}^{2+}$ channel antagonists (Becker et al., 2008; Ritz et al., 2010; Pasternak et al., 2012). The voltage gated L-type $\mathrm{Ca}^{2+}$ channel inhibitor isradipine that blocks $\mathrm{Ca}^{2+}$ entry can protect SNc neurons in mouse models of PD (Ilijic et al., 2011). A phase III clinical trial of isradipine is in progress to evaluate if it can also benefit PD patients (Liss and Striessnig, 2019). However, the relationship between serum calcium and PD has not been well recognized by observational studies. Here, we investigated the genetic associations of serum calcium content with PD risk using the Mendelian randomization method, and the results were negative. Our results are comparable to the previous epidemiological reports, demonstrating that calcium supplementation was not associated with PD risk (Chen et al., 2007; Mischley et al., 2017). The relationship between altered 
serum calcium content and PD reported by a few observational studies might be mediated by some confounding factors such as daily physical activity. In conclusion, our results did not support the supplementation of calcium for preventing PD in the general population. Future clinical trials regarding this topic may fail to produce positive benefits.

Mendelian randomization research has many advantages. Firstly, the study can use the large-scale serum calcium and PD GWAS datasets to provide enough power, thereby detecting genetic associations of serum calcium content with PD. Secondly, these serum calcium and PD GWAS datasets were all of European descent, reducing population stratification effects. Thirdly, various independent genetic variants were used as instrument variants to decrease linkage disequilibrium effects. Fourthly, multiple methods were carried out for a comprehensive pleiotropy analysis to rule out two genetic variants related to potential confounding factors. Causal inference results should not depend on a single method. A previous study indicated that if the results were confirmed using a variety of methods, the causal findings might be more reliable, especially if these methods are based on different assumptions (Burgess et al., 2017). Therefore, we chose 4 different methods to test causal effects in this present study. The application of different methods under different situations may affect the results of the causal effect analysis. Specifically, when investigating whether there is a dose-response relation between the genetic instruments and their outcomes, the MR-Egger method is more effective than the IVW or weightedmedian methods. However, when exploring the possible effects of outlying genetic variants on causality, the IVW estimates may be less severely affected by other factors compared to the MR-Egger estimate (Burgess and Thompson, 2017). In practical applications, it is recommended to use a series of sensitivity analysis methods. All these mentioned advantages could protect against violations of Mendelian randomization assumptions.

Meanwhile, this Mendelian randomization trial also has some limitations. Firstly, the scale of PD GWAS dataset used by us was small, with 8,477 individuals (Pankratz et al., 2012; Liu et al., 2017). In 2014, Nalls et al. (2014) conducted a large-scale GWAS meta-analysis containing 13708 PD cases and 95,282 controls from 15 independent GWAS datasets in European. However, this dataset was not publicly available, so our trial needs to be repeated when this dataset is available. Secondly, population stratification was not absolutely excluded due to its impacts on the assessment. Thirdly, the genetic relationship between serum calcium content and PD might be different due to the various ethnicities and ancestries, so the genetic relationship needs to be further assessed in other ethnicities and ancestries. However, other meta-analyses, such as meta-analysis based on the Bayesian method (Moreno et al., 2014), have also been used to test this kind of relationship. We will further conduct this meta-analysis in the next study to explore the correlation between serum calcium levels and PD and compared it with our Mendelian randomized analysis. Ultimately, there were some difficulties calculating the false positive rates of different methods and we indeed need to address these concerns. We will examine all the concerns mentioned above in future research.
In conclusion, our data indicated that elevated serum calcium levels were not causally related to PD risk. Hence, calcium supplementation might not contribute to reducing PD risk.

\section{DATA AVAILABILITY STATEMENT}

Publicly available datasets were analyzed in this study. This data can be found here: https://www.ebi.ac.uk/gwas/.

\section{AUTHOR CONTRIBUTIONS}

YW and LG designed the study, analyzed the data, and wrote the first draft of the manuscript. PC revised the manuscript. WL, HL, and PC collected the data and provided technological support. WJ conceived the study, formulated the research concept, and revised the manuscript. NZ provided suggestions for the study design, revision and proof of the manuscript. All authors contributed to the interpretation of the results and critical revision of the manuscript for important intellectual content and approved the final version of the manuscript.

\section{FUNDING}

This work was supported by the National Natural Science Foundation of China (Nos. 81801197 and 81870954 to WJ) and the Natural Science Foundation of Tianjin (No. 19JCQNJC10500 to $\mathrm{WJ})$.

\section{ACKNOWLEDGMENTS}

We thank Pankratz and colleagues for the PD GWAS summary results data. We also thank the Global Lipids Genetic Consortium (GLGC), International Consortium of Blood Pressure (ICBP) consortium, DIAbetes Genetics Replication and Meta-analysis (DIAGRAM) Consortium, Genetic Investigation of ANthropometric Traits (GIANT) consortium, Tobacco and Genetics Consortium (TGC), Alcohol Genome-Wide Association (AlcGen), and Cohorts for Heart and Aging Research in Genomic Epidemiology Plus (CHARGE +) Consortia, Global Urate Genetics Consortium (GUGC) and Ukbiobank for other GWAS datasets. We also thank Dr. Junwei Hao for the help in the study design and manuscript drafting.

\section{SUPPLEMENTARY MATERIAL}

The Supplementary Material for this article can be found online at: https://www.frontiersin.org/articles/10.3389/fgene.2020. 00824/full\#supplementary-material 
FILE S1 | Methods to measure serum calcium levels in the original study.

FILE S2 | $P$-values for associations of 8 calcium-associated genetic variants with known PD risk factors.

FILE S3 | Individual causal estimates from each of serum calcium genetic variants and PD using different methods in Discovery PD GWAS dataset.

\section{REFERENCES}

Abou-Raya, S., Helmii, M., and Abou-Raya, A. (2009). Bone and mineral metabolism in older adults with Parkinson's disease. Age Ageing 38, 675-680. doi: 10.1093/ageing/afp137

Ascherio, A., and Schwarzschild, M. A. (2016). The epidemiology of Parkinson's disease: risk factors and prevention. Lancet Neurol. 15, 1257-1272. doi: 10. 1016/s1474-4422(16)30230-7

Becker, C., Jick, S. S., and Meier, C. R. (2008). Use of antihypertensives and the risk of Parkinson disease. Neurology 70, 1438-1444. doi: 10.1212/01.wnl. 0000303818.38960 .44

Bowden, J., Davey Smith, G., and Burgess, S. (2015). Mendelian randomization with invalid instruments: effect estimation and bias detection through Egger regression. Int. J. Epidemiol. 44, 512-525. doi: 10.1093/ije/dyv080

Bowden, J., Davey Smith, G., Haycock, P. C., and Burgess, S. (2016). Consistent estimation in mendelian randomization with some invalid instruments using a weighted median estimator. Genet. Epidemiol. 40, 304-314. doi: 10.1002/gepi. 21965

Brion, M. J., Shakhbazov, K., and Visscher, P. M. (2013). Calculating statistical power in Mendelian randomization studies. Int. J. Epidemiol. 42, 1497-1501. doi: 10.1093/ije/dyt179

Burgess, S., Bowden, J., Fall, T., Ingelsson, E., and Thompson, S. G. (2017). Sensitivity analyses for robust causal inference from mendelian randomization analyses with multiple genetic variants. Epidemiology 28, 30-42. doi: 10.1097/ ede.0000000000000559

Burgess, S., and Thompson, S. G. (2011). Avoiding bias from weak instruments in Mendelian randomization studies. Int. J. Epidemiol. 40, 755-764. doi: 10.1093/ ije/dyr036

Burgess, S., and Thompson, S. G. (2017). Interpreting findings from Mendelian randomization using the MR-Egger method. Eur. J. Epidemiol. 32, 377-389. doi: 10.1007/s10654-017-0255-x

Chen, H., O'reilly, E., Mccullough, M. L., Rodriguez, C., Schwarzschild, M. A., Calle, E. E., et al. (2007). Consumption of dairy products and risk of Parkinson's disease. Am. J. Epidemiol. 165, 998-1006.

Cheng, L., Zhuang, H., Ju, H., Yang, S., Han, J., Tan, R., et al. (2019). Exposing the causal effect of body mass index on the risk of type 2 diabetes mellitus: a mendelian randomization study. Front. Genet. 10:94. doi: 10.3389/fgene.2019. 00094

Cheng, L., Zhuang, H., Yang, S., Jiang, H., Wang, S., and Zhang, J. (2018). Exposing the causal effect of $\mathrm{C}$-reactive protein on the risk of type 2 diabetes mellitus: a mendelian randomization study. Front. Genet. 9:657. doi: 10.3389/fgene.2018. 00657

Dale, C. E., Fatemifar, G., Palmer, T. M., White, J., Prieto-Merino, D., Zabanch, D., et al. (2017). Causal associations of adiposity and body fat distribution with coronary heart disease, stroke subtypes, and type 2 diabetes mellitus: a mendelian randomization analysis. Circulation 135, 2373-2388. doi: 10.1161/ circulationaha. 116.026560

Emdin, C. A., Khera, A. V., Natarajan, P., Klarin, D., Zekavat, S. M., Hsiao, A. J., et al. (2017). Genetic association of waist-to-hip ratio with cardiometabolic traits, type 2 diabetes, and coronary heart disease. JAMA 317, 626-634.

Gibrat, C., Saint-Pierre, M., Bousquet, M., Levesque, D., Rouillard, C., and Cicchetti, F. (2009). Differences between subacute and chronic MPTP mice models: investigation of dopaminergic neuronal degeneration and alphasynuclein inclusions. J. Neurochem. 109, 1469-1482. doi: 10.1111/j.1471-4159. 2009.06072.x

Hu, Y., Zhao, T., Zang, T., Zhang, Y., and Cheng, L. (2018). Identification of Alzheimer's disease-related genes based on data integration method. Front. Genet. 9:703. doi: 10.3389/fgene.2018.00703
FILE S4 | Individual causal estimates from each of serum calcium genetic variants and PD using different methods in Validation PD GWAS dataset.

FILE S5 | Forest plot for MR estimates about the association between genetically increased serum calcium levels and PD in Discovery PD GWAS dataset.

FILE S6 | Forest plot for MR estimates about the association between genetically increased serum calcium levels and PD in Validation PD GWAS dataset.

Ilijic, E., Guzman, J. N., and Surmeier, D. J. (2011). The L-type channel antagonist isradipine is neuroprotective in a mouse model of Parkinson's disease. Neurobiol. Dis. 43, 364-371. doi: 10.1016/j.nbd.2011.04.007

Jiang, Q., Hu, Y., Jin, S., and Liu, G. (2018). Genetically increased serum calcium levels reduce Alzheimer's disease risk. BioRxiv [Preprint]. doi: 10.1101/255059

LaFerla, F. M. (2002). Calcium dyshomeostasis and intracellular signalling in Alzheimer's disease. Nat. Rev. Neurosci. 3, 862-872. doi: 10.1038/nrn960

Larsson, S. C., Burgess, S., and Michaelsson, K. (2017). Association of genetic variants related to serum calcium levels with coronary artery disease and myocardial infarction. JAMA 318, 371-380.

Liss, B., and Striessnig, J. (2019). The potential of L-type calcium channels as a drug target for neuroprotective therapy in Parkinson's disease. Annu. Rev. Pharmacol. Toxicol. 59, 263-289. doi: 10.1146/annurev-pharmtox-010818021214

Liu, G., Bao, X., Jiang, Y., Liao, M., Jiang, Q., Feng, R., et al. (2015a). Identifying the association between Alzheimer's disease and Parkinson's disease using genome-wide association studies and protein-protein interaction network. Mol. Neurobiol. 52, 1629-1636. doi: 10.1007/s12035-014-8946-8

Liu, G., Bao, X., and Wang, R. (2015b). Expression quantitative trait loci regulate HNF4A and PTBP1 expression in human brains. Proc. Natl. Acad. Sci. U.S.A. 112:E3975.

Liu, G., Jin, S., and Jiang, Q. (2019). Interleukin-6 receptor and inflammatory bowel disease: a mendelian randomization study. Gastroenterology 156, 823-824. doi: 10.1053/j.gastro.2018.09.059

Liu, G., Liu, Y., Jiang, Q., Jiang, Y., Feng, R., Zhang, L., et al. (2016). Convergent genetic and expression datasets highlight TREM2 in Parkinson's disease susceptibility. Mol. Neurobiol. 53, 4931-4938. doi: 10.1007/s12035-015-9416-7

Liu, G., Zhao, Y., Jin, S., Hu, Y., Wang, T., Tian, R., et al. (2018). Circulating vitamin E levels and Alzheimer's disease: a Mendelian randomization study. Neurobiol. Aging 72, 189.e1-189.e9. doi: 10.1016/j.neurobiolaging.2018.08.008

Liu, J., Zhou, X. P., Zhang, L., Zhang, Q., Liu, C. F., and Luo, W. F. (2016). [Correlation analysis of serum calcium level and cognition in the patients with Parkinson's disease]. Zhonghua Yi Xue Za Zhi 96, 3284-3288.

Liu, J. Z., Erlich, Y., and Pickrell, J. K. (2017). Case-control association mapping by proxy using family history of disease. Nat. Genet. 49, 325-331. doi: 10.1038/ng. 3766

Manousaki, D., Paternoster, L., Standl, M., Moffatt, M. F., Farrall, M., Bouzigon, E., et al. (2017). Vitamin D levels and susceptibility to asthma, elevated immunoglobulin E levels, and atopic dermatitis: a Mendelian randomization study. PLoS Med. 14:e1002294. doi: 10.1371/journal.pmed.1002294

Meamar, R., Maracy, M., Chitsaz, A., Ghazvini, M. R., Izadi, M., and Tanhaei, A. P. (2013). Association between serum biochemical levels, related to bone metabolism and Parkinson's disease. J. Res. Med. Sci. 18, S39-S42.

Mischley, L. K., Lau, R. C., and Bennett, R. D. (2017). Role of diet and nutritional supplements in Parkinson's disease progression. Oxid. Med. Cell. Longev. 2017:6405278.

Mokry, L. E., Ross, S., Ahmad, O. S., Forgetta, V., Smith, G. D., Goltzman, D., et al. (2015). Vitamin D and risk of multiple sclerosis: a mendelian randomization study. PLoS Med. 12:e1001866. doi: 10.1371/journal.pmed.1001866

Moreno, E., Vazquez-Polo, F. J., and Negrin, M. A. (2014). Objective Bayesian meta-analysis for sparse discrete data. Stat. Med. 33, 3676-3692. doi: 10.1002/ sim.6163

Nalls, M. A., Pankratz, N., Lill, C. M., Do, C. B., Hernandez, D. G., Saad, M., et al. (2014). Large-scale meta-analysis of genome-wide association data identifies six new risk loci for Parkinson's disease. Nat. Genet. 46, 989-993.

Nelson, C. P., Hamby, S. E., Saleheen, D., Hopewell, J. C., Zeng, L., Assimes, T. L., et al. (2015). Genetically determined height and coronary artery disease. N. Engl. J. Med. 372, 1608-1618. 
O’Seaghdha, C. M., Wu, H., Yang, Q., Kapur, K., Guessous, I., Zuber, A. M., et al. (2013). Meta-analysis of genome-wide association studies identifies six new Loci for serum calcium concentrations. PLoS Genet. 9:e1003796. doi: 10.1371/ journal.pgen.1003796

Pankratz, N., Beecham, G. W., Destefano, A. L., Dawson, T. M., Doheny, K. F., Factor, S. A., et al. (2012). Meta-analysis of Parkinson's disease: identification of a novel locus, RIT2. Ann. Neurol. 71, 370-384. doi: 10.1002/ana.22687

Pasternak, B., Svanstrom, H., Nielsen, N. M., Fugger, L., Melbye, M., and Hviid, A. (2012). Use of calcium channel blockers and Parkinson's disease. Am. J. Epidemiol. 175, 627-635.

Ritz, B., Rhodes, S. L., Qian, L., Schernhammer, E., Olsen, J. H., and Friis, S. (2010). L-type calcium channel blockers and Parkinson disease in Denmark. Ann. Neurol. 67, 600-606.

Saad, M., Lesage, S., Saint-Pierre, A., Corvol, J. C., Zelenika, D., Lambert, J. C., et al. (2011). Genome-wide association study confirms BST1 and suggests a locus on $12 \mathrm{q} 24$ as the risk loci for Parkinson's disease in the European population. Hum. Mol. Genet. 20, 615-627. doi: 10.1093/hmg/ddq497

Schapira, A. H. (2013). Calcium dysregulation in Parkinson's disease. Brain 136, 2015-2016. doi: 10.1093/brain/awt180

Sun, W., Han, Y., Yang, S., Zhuang, H., Zhang, J., Cheng, L., et al. (2019). The assessment of interleukin-18 on the risk of coronary heart disease. Med. Chem. 16.

Tillmann, T., Vaucher, J., Okbay, A., Pikhart, H., Peasey, A., Kubinova, R., et al. (2017). Education and coronary heart disease: mendelian randomisation study. BMJ 358:j3542. doi: 10.1136/bmj.j3542
Verbanck, M., Chen, C. Y., Neale, B., and Do, R. (2018). Detection of widespread horizontal pleiotropy in causal relationships inferred from Mendelian randomization between complex traits and diseases. Nat. Genet. 50, 693-698. doi: 10.1038/s41588-018-0099-7

Yavorska, O. O., and Burgess, S. (2017). MendelianRandomization: an R package for performing Mendelian randomization analyses using summarized data. Int. J. Epidemiol. 46, 1734-1739. doi: 10.1093/ije/dyx034

Zhuang, H., Han, J., Cheng, L., and Liu, S. L. (2019a). A positive causal influence of IL-18 levels on the risk of T2DM: a mendelian randomization study. Front. Genet. 10:295. doi: 10.3389/fgene.2019.00295

Zhuang, H., Zhang, Y., Yang, S., Cheng, L., and Liu, S. L. (2019b). A mendelian randomization study of infant length and type 2 diabetes mellitus risk. Curr. Gene Ther. 19, 224-231.

Conflict of Interest: The authors declare that the research was conducted in the absence of any commercial or financial relationships that could be construed as a potential conflict of interest.

Copyright (c) 2020 Wang, Gao, Lang, Li, Cui, Zhang and Jiang. This is an openaccess article distributed under the terms of the Creative Commons Attribution License (CC BY). The use, distribution or reproduction in other forums is permitted, provided the original author(s) and the copyright owner(s) are credited and that the original publication in this journal is cited, in accordance with accepted academic practice. No use, distribution or reproduction is permitted which does not comply with these terms. 